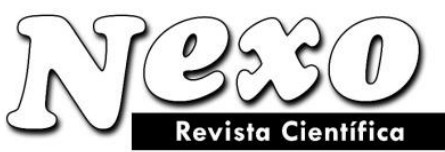

Vol. 34, No. 02, pp. 906-915/Junio 2021
ISSN-E 1995-9516

Universidad Nacional de Ingeniería COPYRIGHT @ (UNI). TODOS LOS DERECHOS RESERVADOS http://revistas.uni.edu.ni/index.php/Nexo https://doi.org/10.5377/nexo.v34i02.11616

\title{
Alternative fractional derivative operator on non-newtonian calculus and its approaches
}

\section{Operador derivado fraccional alternativo sobre cálculo no newtoniano y sus enfoques}

\author{
Mohammad Momenzadeh ${ }^{1}$, Sajedeh Norozpour ${ }^{2 *}$ \\ ${ }^{1}$ Department of Mathematics, Near East University, TRNC, via Mersin 10, Turkey. \\ ${ }^{2}$ Department of Civil Engineering, Istanbul Gelisim University, Istanbul, Turkey. \\ *snorozpour@gelisim.edu.tr
}

(recibido/received: 25-enero-2021; aceptado/accepted: 29-abril-2021)

\begin{abstract}
Nowadays, study on fractional derivative and integral operators is one of the hot topics of mathematics and lots of investigations and studies make their attentions in this field. Most of these concerns raised from the vast application of these operators in study of phenomena's models. These operators interpreted by Newtonian calculus, however different types of calculi are existed and we introduce the fractional derivative operators focused on Bi-geometric calculus and also their fractional differential equations are studied.
\end{abstract}

Keywords: Non-Newtonian calculi, Bi-geometric calculus, Hadamard fractional integral and derivative operators.

\section{RESUMEN}

Hoy en día, el estudio de operadores fraccionarios derivados e integrales es uno de los temas candentes de las matemáticas y muchas investigaciones y estudios centran su atención en este campo. La mayoría de estas preocupaciones surgieron de la vasta aplicación de estos operadores en el estudio de modelos de fenómenos. Estos operadores interpretados por el cálculo newtoniano, sin embargo, existen diferentes tipos de cálculos y se introducen los operadores de derivada fraccionaria enfocados en el cálculo Bigeométrico y también se estudian sus ecuaciones diferenciales fraccionarias.

Palabras clave: Cálculos no newtonianos, Cálculo bi-geométrico, Operadores de derivadas e integrales fraccionarias de Hadamard. 


\section{INTRODUCTION}

Since Newton and Leibnitz introduced modern calculus, many calculi have been created with different aspects. In the 60th decad an underlying idea was given by Michael Grossman and Robert Katz for creating different presentations of Newtonian calculus. The idea based on making the homeomorphisem from real line to $I=(0, \infty)$ that is denoted by $y=\alpha(x)$ which leads to the new defnitions of algebraic operators. On the basis of these operator, it is possible to set two calculi that is called $\alpha$-calculus and bi $\alpha$ calculus. In the existing literature, we consider the bi $\alpha$-calculus that leads to the different derivative and integral in comparison by $\alpha$-calculus. Different functions as $y=\alpha(x)$ lead to different calculi, specificly the case $\alpha(x)=\exp (x)$ was developed in many literatures and its properties were investigated. We list arithmetics $\alpha$-operators and their corresponding specific values for $\alpha(x)=\exp (x)$ which form a field on $I$ and can isometrically transfer the real line to $I$ (Momenzadeh, 2021).

$$
\begin{gathered}
a \bigoplus_{\alpha} b=\alpha\left(\alpha^{-1}(a)+\alpha^{-1}(b)\right) \rightarrow a \bigoplus_{\text {exp }} b=a b \\
a \Theta_{\alpha} b=\alpha\left(\alpha^{-1}(a)-\alpha^{-1}(b)\right) \rightarrow a \Theta_{\text {exp }} b=a / b \\
a \bigotimes_{\alpha} b=\alpha\left(\alpha^{-1}(a) \cdot \alpha^{-1}(b)\right) \rightarrow a \bigotimes_{\text {exp }} b=a^{\ln b} \\
a \oslash_{\alpha} b=\alpha\left(\alpha^{-1}(a) / \alpha^{-1}(b)\right) \rightarrow a \oslash_{\text {exp }} b=\sqrt[\ln b]{a}
\end{gathered}
$$

These definitions of arithmetics $\alpha$-operators inspired the derivative and integral of non-Newtonian calculus that their definitions and corresponding relations to Newtonian calculus are as follow (Ezzat, 2010)

$$
\begin{gathered}
f^{\widehat{\alpha}}(x)=\lim _{y \rightarrow x}\left(\left(f(y) \Theta_{\alpha} f(x)\right) \oslash_{\alpha}\left(y \Theta_{\alpha} x\right)\right)=\alpha\left(\frac{\alpha^{-1}(f(x)) \prime}{\alpha^{-1}(x) \prime}\right) \\
\int_{a}^{b} f(x) d^{\widehat{\alpha}}(x) \\
=\lim _{n \rightarrow \infty}{ }_{i=1}^{n} \bigoplus_{\alpha} f\left(c_{i}\right) \otimes_{\alpha}\left(x_{i+1} \Theta_{\alpha} x_{i}\right) \\
=\alpha\left(\int_{a}^{b} \alpha^{-1}(f(x)) \alpha^{-1}(x)^{\prime} d x\right) .
\end{gathered}
$$

For more than 60 years that the concepts of non-Newtonian calculus were stablished, few articles have been published with the main concerns of this calculus and the structure of these calculi were studied. At first glance, it seems that the simple homeomorphism cannot make a significant difference on the concepts. However, the study of fractional derivative and integral shows the interesting relations and significant differences which are concerned in this article. The tools of fractional calculus have played a significant role in improving the modeling techniques for several real-world problems and we can Önd trace of this operator in many articles. Fractional derivative simply can be considered as the derivative of any order and seems that its origin return back to a letter from Leibniz to líHopital in 1695. There are many approaches to the fractional integral operator as making the differential equation and finding the operator as an extension of solution, or using Cauchy iterated integral. Indeed, we defined the fractional integral operator for non-Newtonian calculi in the aid of Cauchy iterated integral by applying (3) in the chain of integration and reach to this definition (Ezzat, 2010).

$$
\begin{gathered}
\alpha(x) I_{x}^{n} f(x)=\int_{a}^{x} \int_{a}^{x_{1}} \ldots \int_{a}^{x_{n-1}} f\left(x_{n}\right) d^{\widehat{\alpha}} x_{n} d^{\widehat{\alpha}} x_{n-1} \ldots d^{\widehat{\alpha}} x_{1} \Rightarrow \\
\underset{{ }_{a} I_{x}^{\mu}}{\alpha(x)}=\alpha\left(\frac{1}{\Gamma(\mu)} \int_{a}^{x}\left(\alpha^{-1}(x)-\alpha^{-1}(s)\right)^{\mu-1} \alpha^{-1}(f(s)) \alpha^{-1}(s)^{\prime} d s\right) .
\end{gathered}
$$


To define fractional derivative in non-Newtonian calculus, one may use Lagrangeís rule for differential operators. Computing $n$-th order derivative over the integral of order $(n-\beta)$ the $\beta$ order derivative is obtained where $n$ is the smallest integer greater than $\beta$ This leads us to the following definition (Pandey \& Holm, 2016).

$$
{ }_{a}^{\alpha(x)} D_{x}^{\beta} f(x)=\left(\frac{d^{\widehat{\alpha}}}{d x^{\widehat{\alpha}}}\right)^{n}{ }_{a}^{\alpha(x)} D_{x}^{-(n-\beta)} f(x)=\left(\frac{d^{\widehat{\alpha}}}{d x^{\widehat{\alpha}}}\right)^{n}{ }_{a}^{\alpha(x)} I_{x}^{n-\beta} f(x)
$$

We will study the fractional differential equation (5) in specific case i.e. $\alpha(x)=\exp (x)$ in the following sections, although related fractional differential equations and their applications in describing real-world problems will be introduced (Pandey \& Holm, 2016).

\section{DERIVATIVE OPERATOR AND ELASTICITY ON BI-GEOMETRIC CALCULUS}

Out of all examples of non-Newtonian calculus, one of the brilliant cases occurred when we substitute $\exp (x)$ instead of $\alpha(x)$ which is called bi-geometric calculus. In this case, the derivative and integral can be defined as (Letelier \& Stockle, 2021).

$$
\begin{gathered}
f^{\circ}(x)=\lim _{y \rightarrow x} \sqrt[\ln \left(\frac{y}{x}\right)]{\frac{f(y)}{f(x)}}=\lim _{h \rightarrow 0}\left(\frac{f(x+h)}{f(x)}\right)^{\left(\frac{1}{h}\right)}=\exp \left(x \frac{f(x) \prime}{f(x)}\right) \\
\int_{a}^{b} f(x)^{d x}=\prod_{i=0}^{\infty}\left(f\left(x_{i}\right)\right)^{\ln \left(\frac{x_{i+1}}{x_{i}}\right)} \\
=\exp \left(\int_{a}^{b} \ln (f(x)) \frac{d x}{x}\right) \\
\text { where the } x_{i} \text { forms the partion for }[a, b]
\end{gathered}
$$

We denote the derivative operator of bi-geometric calculus by $D^{\circ}(f)=f^{\circ}$ and It is easy to see that ${ }^{\circ}$ derivative and Newtonian derivative are coexist; that is, for positive real function if either exists then so does the other. There are many interesting properties of this operator which were studied successfully. For instance, ${ }^{\circ}$-derivative of power function is equal to their ${ }^{\circ}$ - slope and is constant. In addition, the operator $D^{\circ}$ is scale-free; that is, $D^{\circ}$ is independent of the scale or unit which are used for domain and range of the function. In a pedagogical literature Segel (1972) discusses the necessity and advantages for nondimensionalisation and scaling in general with several practical examples and this derivative gives the oportunity of making the easier model with no dimension.In the study of economy, the expression at parentheses in (6) is called elasticity and because of that we refer $f^{\circ}(x)$ as resiliency of $f$ at $x$. Let us describe this economical concept precisely and see how can we use this interpretation. Elasticity is defined as a measure of a variableís sensitivity to change in another variable and our concern is elasticity of demand and it is predominantly used to assess the change in consumer demand as a result of a change in a good or serviceís price. This measure is calculated as percentage of change of quantity over the percentage of change in price (Sene, 2018). There is two cases of consideration, when the elasticity is greater than one, demand for good or service is a§ect by price and when it is less than one, buying habits stay about the same. The good example of elasticity which is greater than one, can be assumed as the candy which can be replaced by another sweet products and for the case that elasticity is less than one, can be assumed as insulin medicine which can not be replaced easily. If we refer quantity as a function of price and denote it by $y$ and $x$ respectively, then we can see that (Sene, 2018).

$$
\text { Elasticit of demand }=\frac{\% \text { change of quality }}{\% \text { change in price }}
$$




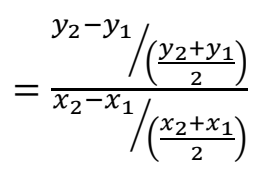

Now by tending $x_{1}$ to $x_{2}$, we reach to $\ln \left(f^{\circ}\left(x_{2}\right)\right)$. Indeed, since the derivative of bi-geometric calculus can be interpreted as exponential of elasticity, the critical value is one, similar to the critical value of Newtonian derivative which is zero. Therefore, the interpretation of derivative in bi-geometric calculus can lead us to another prespective for modelling the real-worlds phenomena. Definitely, the direct usage of this concept can be applied in economic issues and further fractional derivative can be used to make a better curve fitting function, whereas in this literature we apply it for simple population growth model and we use this concept to interpret the growth rate model (Odibat \& Momani, 2008).

The study of population change has a very long history and can be traced back to the work of Leonardo in the excersice of his arithmetic book that involved building a mathematical model for a growing rabbit population. From very begining of study the model for population growth rate where $N(t)$ denotes the population of the speciest at time $t$ and known as the conservation equation of population was introduced as (Odibat \& Momani, 2008).

$$
\frac{d N}{d t}=\text { birth }- \text { death }+ \text { migration }
$$

This simple model of population rate inspired a lot of mathematician to developed their model in the different aspects. The pioneer of them was Malthus who described his model simply as right side of (9) as a constant coeficient of $N(t)$, population. His model was unrealistic but it was the first atempt to establish the mathematical model based on conservation equation of population and clearify that the right side of the equation in (9) should be written as a function of $N(t)$. In the long run of course there must be some adjusment to such exponential growth and at the end Verhulst solved the problem of population increasing by putting the self-limiting process in the equation and introduced the logistic growth in a population in the aid of following equation (Odibat \& Momani, 2008).

$$
\frac{d N}{d t}=r N\left(1-\frac{N}{k}\right) \quad r, k>0
$$

There is a remarkable note that by going forward to the recent time, a lot of model were studied to make more accurate model and give the better approximation. Definitely, we could produce an algebraic expression with a few more parameters and derive some differential equation for which it is the solution and do a better job but then all we would be doing would be curve fitting without increasing our understanding of the actual mechanism governing the phenomenon. The motivation for modelling is to further our understanding of the underlying processes since it is only in this way that we can make justiÖable predictions.Our consider in this article is the Malthus model which is simply described the population growth. However, this discussion can be extended to more complicated and advanced models. Malthus considered the simple model of population with no imigration and assumed the birth and death terms as a proportion of population which can be written in terms of Newtonian calculus as follow: (Ghalib et. al, 2020).

$$
\frac{d N}{d t}=b N-d N \rightarrow N(t)=N_{0} e^{(b-d) t}
$$

Here $b$ and $d$ are positive constant and $N_{0}$ denotes the initial value of population. In this model, if the birth factor $b$ is greater than death factor $d$, then the population grows exponentially while $b<d$ implies that it 
dies out. In addition, the model is not dimension free; that is the constants $b$ and $d$ have the dimension (time $)^{-1}$ and both side of the equation have dimension of $N t^{-1}$. If we construct ${ }^{\circ}$-derivative of population, then we can see the model as (Ghalib et. al, 2020).

$$
D^{\circ}(N)=e^{(b-d) t}
$$

This equation expresses the model with a simple relation between the single ${ }^{\circ}$-derivative and exponential function and has the nondimensional form. Indeed, the model declare that elasticity of population is linearly equal to $(b-d) t$ and by going forward through the time, population from inelastic stage changes to greater elasticity. By the other words, when the time is increasing, the population rapidly affected by changing the time and because of that the model is not realistic. This preliminary example shows that how the bi-geometric calculus could be used to describe the model and this interpretation gives a new outview to population modelling wheares all kind of calculi are isometrically the same (Ghalib et. al, 2020).

\section{FRACTIONAL DERIVATIVE ON BI-GEOMETRIC CALCULUS}

The fractional integral operator on bi-geometric calculus can be written by substituting $\exp (x)$ instead of $\alpha(x)$ at (4) which is (Alotta et. al, 2019).

$$
\left.{ }_{a}^{\exp (x)} I_{x}^{\mu} f(x)=\exp \left(\frac{1}{\Gamma(\mu)} \int_{a}^{x}\left(\ln \left(\frac{x}{s}\right)\right)\right)^{\mu-1} \ln f(s) \frac{d s}{s}\right)
$$

The expression in the braces at (11) can be considered as the Hadamard integral operator on $\ln f(x)$ which is defined as (Alotta et. al, 2019).

$$
{ }_{a}^{H} J_{x}^{\mu} f(x)=\frac{1}{\Gamma(\mu)} \int_{a}^{x}\left(\ln \left(\frac{x}{s}\right)\right)^{\mu-1} f(s) \frac{d s}{s},
$$

Let us review some properties of fractional Hadamard operators first. Nowaday we can see the vast usage of fractional differential equations in improving the models of several phenomena. However, most of the work on the topic is based on Riemann-Liouville, and Caputo-type fractional differential equations and contribution of Hadamard fractional operators is insignificant which is apeared side by side to RiemannLiouville and Caputo derivatives in the literature and is the fractional derivative due to Hadamard, introduced in 1892. Hadamard fractional integral can be formulated from the following Cauchy iterated (Siddique \& Bukhari, 2020).

$$
{ }_{a}^{H} J_{x}^{\mu} f(x)=\int_{a}^{x} \frac{d t_{1}}{t_{1}} \int_{a}^{t_{1}} \frac{d t_{2}}{t_{2}} \ldots \int_{a}^{t_{n-1}} f\left(t_{n}\right) \frac{d t_{n}}{t_{n}}=\frac{1}{(n-1) !} \int_{a}^{x}\left(\ln \left(\frac{x}{s}\right)\right)^{n-1} f(s) \frac{d s}{s} .
$$

The Hadamard fractional derivative is inspired by a fractional power of the form $\delta^{v}=\left(x \frac{d}{d x}\right)^{v}$ which is invariant with respect to dilation on the whole axis and fully compatible with bi-geometric calculus. The left-sided Hadamard fractional derivative operator is defined as (Siddique \& Bukhari, 2020).

$$
{ }_{a}^{H} D_{x}^{\mu} f(x)=\delta_{a}^{n H} D_{x}^{n-\mu} f(x)=\left(x \frac{d}{d x}\right)^{n} \frac{1}{\Gamma(n-\mu)} \int_{a}^{x}\left(\ln \left(\frac{x}{s}\right)\right)^{n-\mu-1} f(s) \frac{d s}{s}
$$


The Hadamard fractional integral and derivative operators have the semigroup properties for $f \in L^{p}(a, b)$ as (pp:114 property 2.26) (Siddique \& Bukhari, 2020).

$$
\begin{gathered}
{ }_{a}^{H} J_{x}^{\mu}\left({ }_{a}^{H} I_{x}^{v} f(x)\right)={ }_{a}^{H} J_{x}^{\mu+v} f(x) \quad \mu, v>0 \\
{ }_{a}^{\alpha(x)} D_{x}^{\mu}\left({ }_{a}^{H} J_{x}^{v} f(x)\right)={ }_{a}^{H} D_{x}^{\mu-v} \quad v>0
\end{gathered}
$$

Kilbas et al. (n.d) studied the Cauchy problem for the nonlinear Hadamard fractional differential equation of complex order $v \in \mathbb{C}$ where $\operatorname{Re}(v)>0$ as following (Siddique \& Bukhari, 2020).

$$
\begin{array}{lc}
{ }_{a}^{H} D_{x}^{v} y(x)=f[x, y(x)] & a \leq x \leq b \\
{ }_{a}^{H} D_{x}^{v-k} y(a)=b_{i} \in \mathbb{C} & (k=1,2, \ldots n)
\end{array}
$$

Where for non-positive integer $n$ is defined as $\lfloor\operatorname{Re}(v)\rfloor+1$ and for $n \in \mathbb{N}, n=v$. It was shown that the solution of this problem can be written as a Volterra integral equation of the second kind of the form:

$$
y(x)=\sum_{i=1}^{n} \frac{b_{i}}{\Gamma(n-i+1)}\left(\ln \left(\frac{x}{a}\right)\right)^{v-i}+\frac{1}{\Gamma(v)} \int_{a}^{x}\left(\ln \left(\frac{x}{s}\right)\right)^{v-1} f[s, y(s)] \frac{d s}{s}
$$

form of solution (16) is useful when we apply the successive approximation with the given initial values. Indeed, to solve this problem for individual cases of $f[x, y(x)]$ which veriÖes the existance conditions by having lipschitz conditions, successive approximation method were used frequently.[9]In the process of the solution we face with different types of Mittag-Leffler functions that the classic form is known as (Kadak \& Özlük, 2015).

$$
E_{v}(z):=\sum_{k=0}^{\infty} \frac{z^{k}}{\Gamma(v k+1)} \quad(z, v \in \mathbb{C} ; \operatorname{Re}(v)>0) .
$$

the simplest and for applications most important generalizations of the Mittag- Leffler function, namely the two-parametric Mittag-Leffler function were investigated by Humbert and Agraval separately in 1953 and can be written as (Kadak \& Özlük, 2015).

$$
E_{v, \tau}(z):=\sum_{k=0}^{\infty} \frac{z^{k}}{\Gamma(v k+\tau)}(z, v, \tau \in \mathbb{C} ; \operatorname{Re}(v)>0) .
$$

Indeed, more general case is apeared in the solution of integral equation (16), where $f[x, y(x)]=$ $(x-a)^{\eta} y(x)$ and was introduced by Kilbas et al. (n.d).

Now, we can define the fractional derivative on bi-geometric calculus by substituting $\exp (x)$ instead of $\alpha(x)$ at (5), which leads to (Kadak \& Özlük, 2015)

$$
\begin{aligned}
{ }_{a}^{\exp (x)} D_{x}^{\beta} f(x) & =\left(\frac{d^{\hat{o}}}{d x^{\circ}}\right)_{a}^{n}{ }_{a}^{\exp (x)} D_{x}^{-(n-\beta)} f(x)=\left(\frac{d^{\hat{o}}}{d x^{\circ}}\right)^{n}{ }_{a}^{\exp (x)} I_{x}^{n-\beta} f(x) \\
& =\left(\frac{d^{\circ}}{d x^{\circ}}\right)^{n} \exp \left(\frac{1}{\Gamma(n-\beta)} \int_{a}^{x}\left(\ln \left(\frac{x}{s}\right)\right)^{n-\beta-1} f(s) \frac{d s}{s}\right)
\end{aligned}
$$


If we assume the derivative operator of Hadamard as $\delta=x \frac{d}{d x}$, then it is easy to see that (Hristov et. al, 2017)

$$
\left(\frac{d^{\hat{o}}}{d x^{\circ}}\right)^{n} y=\exp \left(\delta^{n} \ln (y)\right)
$$

Therefore, we can reduce the fractional derivative of bi-geometric calculus to the following form

$$
{ }_{a}^{\exp (x)} D_{x}^{\beta} f(x)=\exp \left(\left(x \frac{d}{d x}\right)^{n} \frac{1}{\Gamma(n-\beta)} \int_{a}^{x}\left(\ln \left(\frac{x}{S}\right)\right)^{n-\beta-1} f(s) \frac{d s}{s}\right)=\exp \left({ }_{a}^{H} D_{x}^{\mu} \ln f(x)\right)
$$

For instance, let us assume the Particular case where $0<\alpha<1, n=1$ (Hristov et. al, 2017)

$$
\begin{aligned}
\underset{a}{\exp (x)} D_{x}^{\beta} f(x) & =\left(\frac{d^{\hat{o}}}{d x^{\circ}}\right){ }_{a}^{\exp (x)} I_{x}^{1-\beta} f(x)=\exp \left({ }_{a}^{H} J_{x}^{1-\beta} \ln f(x)\right) \\
& =\exp \left(\frac{x \frac{d}{d x}{ }_{a}^{H} J_{x}^{1-\beta} \ln f(x) \exp \left({ }_{a}^{H} J_{x}^{1-\beta} \ln f(x)\right)}{\exp \left({ }_{a}^{H} J_{x}^{1-\beta} \ln f(x)\right)}\right)=\exp \left({ }_{a}^{H} D_{x}^{\beta} \ln f(x)\right)
\end{aligned}
$$

Where ${ }_{a}^{H} D_{x}^{\beta}=\left(x \frac{d}{d x}\right){ }_{a}^{H} J_{x}^{1-\beta}$ Hadamard fractional derivative operator. For instance, let us show that our definition of fractional derivative is compatible by the ${ }^{\circ}$-derivative by an example (Hristov et. al, 2017)

Example 1 Consider ordinary ${ }^{\circ}$-derivative equation as

$$
D^{\circ}(y(x))=y(x)
$$

which is separable differential equation in Newtonian calculus and has a solution as $y(x)=\exp (c x)$. On the other hands corresponding fractional differential equation can be written as (Eldred et. al, 1995)

$$
{ }_{a}^{\exp (x)} D_{x}^{\beta} y(x)=y(x)
$$

that according to our definition is equivalent to Hadamard-type fractional differential equations as ${ }_{a}^{H} D_{x}^{\beta} \ln y(x)=\ln y(x)$ and in the aid of (16) and using succesive approximation the solution in terms of (18) can be written as (pp. 235 Theorem 4.5) (Eldred et. al, 1995)

$$
\ln y=b\left(\ln \left(\frac{x}{a}\right)\right)^{\beta-1}+E_{\alpha, \alpha}\left(\ln \left(\frac{x}{a}\right)\right)^{\beta 1}
$$

We remind that $E_{1,1}(x)=\exp (x)$ and by letting $\beta \rightarrow 1$ We will find the ordinary solution.

As we have seen, derivative of bi-geometric calculus can be considered as resiliancy and with this interpretation, the real-world problems can be remodeled and for getting better curve fitting, the fractional derivative and related differential equation can be obtained. Indeed, several phenomena in the aid of geometric calculus (multiplicative calculus) were studied and related differential equations were established [14] which can be assumed as a good resource of investigation and the separated study. The above example and previous discussions were depended on Hadamard fractional operators and Newtonian 
demonstration of equations and their solutions, but the next lemma is written totally in the bi-geometric symbols (Zhou et. al, 2018).

Lemma. Let $a, \beta>0$ then the following identities hold true

$$
\begin{gathered}
\exp (x){ }_{a}^{\beta} I_{x}^{\beta}\left(\left(t \ominus_{\exp } a\right)^{\circledast \exp (v-1)}\right)(x) \\
=\exp \left(\frac{\Gamma(v)}{\Gamma(v+\beta)}\right) \otimes_{\exp }\left(x \ominus_{\exp } a\right)^{\circledast \exp (\beta+v-1)} \\
\exp (x) D_{x}^{\beta}\left(\left(t \ominus_{\exp } a\right)^{\circledast \exp (v-1)}\right)(x) \\
=\exp \left(\frac{\Gamma(v)}{\Gamma(v-\mu)}\right) \otimes_{\exp }\left(x \ominus_{\exp } a\right)^{\circledast \alpha(\beta-v-1)}
\end{gathered}
$$

Proof. First let us clear the terminology that is used in these identities. According to (1), the exponent of the expression in bi-geometric calculus which is denoted by $\circledast_{\alpha}$ can be defined similar to Newtonian calculus. For instance, we can see that for positive integerís exponent we have (Zhou et. al, 2018):

$$
x^{\circledast n} n=x \otimes_{\alpha} x \ldots \otimes_{\alpha} x=\exp \left((\ln (x))^{n}\right)=x^{(\ln x)^{(n-1)}}
$$

Now let us prove equation (19), the equation (20) can be proved similarly. The prove is straightforward and can be written in the aid of beta function and knowing that $\delta^{n}\left(\ln ^{m} x\right)=\ln ^{m-n} x$ as

$$
\begin{gathered}
\exp (x) I_{x}^{\beta}\left(\left(t \ominus_{\exp } a\right)^{\circledast \exp (v-1)}\right)(x) \\
={ }^{\exp (x)} I_{x}^{\beta}\left(\left(\ln \frac{t}{a}\right)^{v-1}\right)(x) \\
=\exp \left({ }_{a}^{H} J_{x}^{\beta}\left(\left(\ln \frac{t}{a}\right)^{v-1}\right)(x)\right) \\
=\exp \left(\frac{\Gamma(v)}{\Gamma(v+\beta)}\left(\ln \frac{x}{a}\right)^{\beta+v-1}\right) \\
\exp \left(\frac{\Gamma(v)}{\Gamma(v+\beta)}\right) \otimes_{\exp }\left(x \ominus_{\exp } a\right)^{\circledast \exp (v+\beta-1)}
\end{gathered}
$$

It is interesting when we compare it by the identity for Riemann- fractional operator's identities ( $p p .71$ Property 2.1)

$$
\begin{aligned}
& { }_{a} I_{x}^{\beta}\left((t-a)^{v-1}\right)(x)=\frac{\Gamma(v)}{\Gamma(v+\beta)}(x-a)^{\beta+v-1} \\
& { }_{a} D_{x}^{\beta}\left((t-a)^{v-1}\right)(x)=\frac{\Gamma(v)}{\Gamma(v-\beta)}(x-a)^{\beta-v-1}
\end{aligned}
$$

Remark. The derivative of a constant are, in general, not equal to zero and similar to Riemann fractional operator, in the aid of the previous Lemma, it can be seen easily that (Zhou, 2018)

$$
{ }_{a}^{\exp } D_{x}^{\beta}\left(1_{\exp }\right)(x)={ }_{a}^{\exp } D_{x}^{\beta}(e)(x)=\exp \left(\frac{1}{\Gamma(1-\beta)} \ln \left(\frac{x}{a}\right)^{-\beta}\right)
$$

Which for Riemann fractional derivative, 
${ }_{a} D_{x}^{\beta}(1)(x)=\frac{(x-a)^{-\beta}}{\Gamma(1-\beta)}$

In addition, since the Gamma function at zero is not defined we use the direct calculation to see (20) for the values $v=0$ and $0<\beta<1$ as (Zhou et. al, 2018)

$$
\begin{array}{r}
{ }_{a}^{\exp } D_{x}^{\beta}\left(\left(t \ominus_{\exp } a\right)^{\circledast \exp (-1)}\right)(x)=\exp \left({ }_{a}^{H} D_{x}^{\beta}\left(\ln \frac{t}{a}\right)^{-1}(x)\right) \\
=\exp \left(\left(x \frac{d}{d x}\right) \frac{1}{\Gamma(1-\beta)} \int_{a}^{x}\left(\ln \frac{x}{s}\right)^{-\beta}\left(\ln \frac{s}{a}\right)^{-1} \frac{d s}{s}\right)=1
\end{array}
$$

\section{CONCLUSION}

In this paper we introduced the fractional integral and derivative on non-Newtonian calculus. Many real examples of the phenomena were described in the aid of non-Newtonian calculus and new approaches to these problems were considered. In the discussion of fractional operators related to the general function, similar discussion can be assumed. However, this interpretation in nonNewtonian calculus can construct the bridge between these two parts of mathematics. Moreover, the fractional operators on non-Newtonian calculus are totally compatible with the structure of these calculi. This interpretation of fractional calculus as a specific operator in corresponding calculus, lets us determine many results, especially economical debates, as a fractional differential equation in that calculus. As it mentioned in the article, many examples of different aspects such as population problem, economical problems can be well established in terms of non-Newtonian calculus. Plugging the fractional operators to these calculi makes the interpretations clear and many problems and applications could struggle with these things.

\section{REFERENCES}

Momenzadeh, M. (2021, February). On the alternative approach to $\psi$-fractional calculus. In AIP Conference Proceedings (Vol. 2325, No. 1, p. 020020). AIP Publishing LLC.

Ezzat, M. A. (2010). Thermoelectric MHD non-Newtonian fluid with fractional derivative heat transfer. Physica B: Condensed Matter, 405(19), 4188-4194.

Pandey, V., \& Holm, S. (2016). Linking the fractional derivative and the Lomnitz creep law to non-Newtonian time-varying viscosity. Physical Review E, 94(3), 032606.

Letelier, M., \& Stockle, J. (2021). Some applications of extended calculus to non-Newtonian flow in pipes. Journal of the Brazilian Society of Mechanical Sciences and Engineering, 43(2), 114.

Sene, N. (2018). Stokes' first problem for heated flat plate with Atangana-Baleanu fractional derivative. Chaos, Solitons \& Fractals, 117, 68-75.

Odibat, Z. M., \& Momani, S. (2008). An algorithm for the numerical solution of differential equations of fractional order. Journal of Applied Mathematics \& Informatics, 26(1_2), 15-27. 
Ghalib, M. M., Zafar, A. A., Hammouch, Z., Riaz, M. B., \& Shabbir, K. (2020). Analytical results on the unsteady rotational flow of fractional-order non-Newtonian fluids with shear stress on the boundary. Discrete \& Continuous Dynamical Systems-S, 13(3), 683.

Alotta, G., Bologna, E., Failla, G., \& Zingales, M. (2019). A fractional approach to nonNewtonian blood rheology in capillary vessels. Journal of Peridynamics and Nonlocal Modeling, $1(2), 88-96$.

Siddique, I., \& Bukhari, S. M. (2020). Analysis of the effect of generalized fractional Fourier's and Fick's laws on convective flows of non-Newtonian fluid subject to Newtonian heating. The European Physical Journal Plus, 135(1), 1-21.

Kadak, U., \& Özlük, M. (2015, February). Generalized Runge-Kutta method with respect to the non-Newtonian calculus. In Abstract and Applied Analysis (Vol. 2015). Hindawi.

Hristov, J. (2017). Derivation of the fractional Dodson equation and beyond: Transient diffusion with a non-singular memory and exponentially fading-out diffusivity. Progr. Fract. Differ. Appl, $3(4), 1-16$.

Eldred, L. B., Baker, W. P., \& Palazotto, A. N. (1995). Kelvin-Voigt versus fractional derivative model as constitutive relations for viscoelastic materials. AIAA journal, 33(3), 547-550.

Zhou, H. W., Yang, S., \& Zhang, S. Q. (2018). Conformable derivative approach to anomalous diffusion. Physica A: Statistical Mechanics and its Applications, 491, 1001-1013. 